\title{
A Mathematical Model of Communication with Reputational Concerns
}

\author{
Ce Huang, ${ }^{1}$ Yuanyuan Zhang, ${ }^{2}$ and Chong Lai ${ }^{3}$ \\ ${ }^{1}$ School of Public Finance and Taxation, Southwestern University of Finance and Economics, 555 Liutai Avenue, \\ Wenjiang, Chengdu, Sichuan 611130, China \\ ${ }^{2}$ School of Securities and Futures, Southwestern University of Finance and Economics, 555 Liutai Avenue, \\ Wenjiang, Chengdu, Sichuan 611130, China \\ ${ }^{3}$ School of Finance, Southwestern University of Finance and Economics, 555 Liutai Avenue, Wenjiang, Chengdu, Sichuan 611130, China \\ Correspondence should be addressed to Ce Huang; huangce@swufe.edu.cn
}

Received 29 January 2016; Accepted 9 March 2016

Academic Editor: Christos K. Volos

Copyright (c) 2016 Ce Huang et al. This is an open access article distributed under the Creative Commons Attribution License, which permits unrestricted use, distribution, and reproduction in any medium, provided the original work is properly cited.

We investigate a mathematical model where an expert advises a decision maker for two periods. The decision maker is initially unsure about whether the expert is biased or not. After consulting the expert on the decision problem of period one, the decision maker updates belief about the expert's bias and consults the expert on the problem of period two. We find that more information is delivered in the model's first period than in the one-period situation of communication.

\section{Introduction}

This paper studies a mathematical model involving a decision maker and an expert with two periods. In each period, the decision maker needs to make a decision but does not know which one is the best. There is an expert who knows the optimal decisions but may be biased in favor of decisions that are different from the ideal ones of the decision maker. Initially unsure about whether the expert is biased or not, the decision maker consults the expert for the ideal decision in period one. After receiving the expert's advice, the decision maker makes the first-period decision and updates belief about the expert's bias. Then the decision maker consults the expert for the optimal decision in period two. We study whether the expert delivers more information on the optimal decision in period one to the decision maker, compared to the case where the decision maker consults the expert only in period one.

Many situations in real life are captured by the model described above. For instance, a patient usually has less information about his illness than a doctor does. So the former consults the latter. However, the latter may prefer the former to buy expensive medicine or to take medical procedures, even when the patient's disease is mild and these medicine and procedures are unnecessary. The patient is often uncertain about whether the doctor has such preferences. But he can form some belief about the doctor's preferences based on the doctor's former prescriptions. The doctor expects that former prescriptions will affect the patient's belief of the doctor's preferences and influence future communications between the patient and the doctor. Will the doctor give more accurate prescriptions in period one, compared to the case where the doctor advises the patient only in period one?

We find that the expert communicates more information to the decision maker about the optimal decision in period one. This is because if the decision maker believes that the expert is more likely to be unbiased at the end of period one, the expert's payoff will be higher in period two. In period one, the expert engages less in misreporting information, in order to increase the probability that the decision maker believes that the expert is unbiased at the end of period one. As a result, more information is communicated between the expert and the decision maker in period one.

There are several papers that study similar issues as the current paper does. In these papers, the decision maker is uncertain about the expert's bias and the expert is concerned about establishing a reputation for being unbiased. There is another strand of literature studying the case where experts 
observe signals about the state with different accuracies and each expert prefers to be perceived as having accurate information. In Ottaviani and Sørensen [1], it is shown that experts with reputational concerns for having accurate information typically do not wish to tell the truth. In Bourjade and Jullien [2], the expert cannot misreport the information but can conceal the information. In the current paper, the expert is not concerned with establishing a reputation for having accurate information since it is assumed that all experts have perfect information about the state. In Sobel [3], the expert communicates with the decision maker repeatedly. The expert may be biased in favoring a particular decision. In Benabou and Laroque [4], a model similar to the one in Sobel [3] is studied. Li [5] studies the information transmission between the expert and the decision maker through an intermediary. All papers mentioned above assume that the unbiased expert truthfully reports information. In this paper, the unbiased expert may lie in order to enhance his reputation.

The paper that is closest to the current paper is that of Morris [6], who also studies a two-period information transmission model between a decision maker and an expert. It is found that the unbiased expert may send a report different from the observed signal in order to enhance the expert's reputation for being unbiased. Our study is different from that of Morris [6] in the following ways. First, we assume that the expert can perfectly observe the state. Second, there is a continuum of possible states and decisions in the current paper, whereas, in Morris [6], only two states and decisions are possible.

The remaining part of the paper proceeds as follows. Section 2 builds a two-period model of strategic information transmission between a decision maker and an expert. In addition, it characterizes an equilibrium in period two of the model and an equilibrium in period one. Section 3 compares the ex ante expected payoff of the decision maker in period one of the model to that of the decision maker when the decision maker consults the expert in only one period. Section 4 concludes and discusses a direction for future work.

\section{Model}

There are two periods. A decision maker (hereafter DM) needs to take a decision in each period. The decision is to choose a real number in the interval $[0,1]$. In period $t \in\{1,2\}$, after DM takes decision $a_{t} \in[0,1]$, DM receives some payoff, which depends on the underlying state in period $t$ denoted as $s_{t}$. Assume that the payoff of DM in period $t$ is

$$
-\left(a_{t}-s_{t}\right)^{2}
$$

Apparently, the payoff is higher when the decision $a_{t}$ is closer to $s_{t}$. Therefore, the decision that gives DM the highest payoff in period $t$ (hereafter the optimal decision of DM in period $t$ ) is equal to $s_{t}$. However, DM does not know $s_{t}$ except that it is uniformly distributed over $[0,1]$ and $s_{1}$ is distributed independently of $s_{2}$.

There is an expert who observes $s_{t}$ at the beginning of period $t$ (throughout the paper, we use "he" to denote the expert and "she" to denote DM). However, the ideal decision

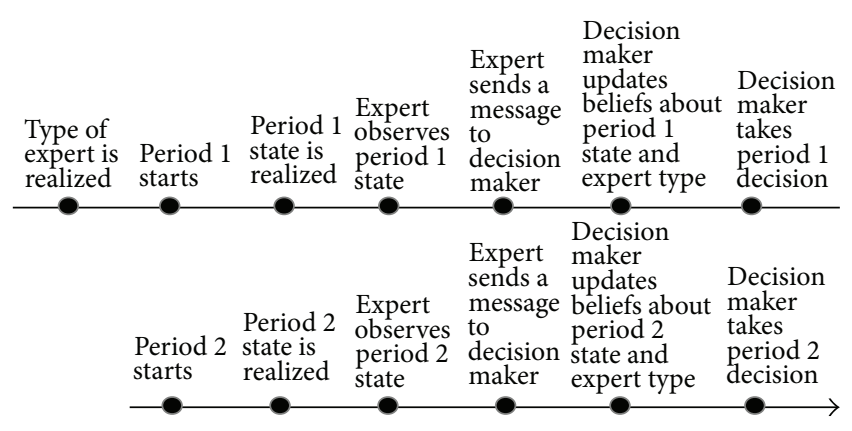

FIGURE 1: Timeline of the two-period communication model.

of the expert may be different from that of DM. We assume that, in period $t$, the payoff received by the expert when DM takes decision $a_{t}$ is

$$
-\left(a_{t}-s_{t}-\beta\right)^{2}
$$

The expert can be of two types. For one type of the expert, $\beta=0$ and the expert's ideal decision in period $t$ is the same as that of DM. We call this type of the expert "unbiased." For the other type, $\beta=b>0$ and the expert's ideal decision is higher than that of DM by $b$. We call this type of the expert "biased." The expert knows his own type, but DM does not. Initially, DM believes that the expert is of either type with equal probabilities. In addition, the expert's type is distributed independently of the state in each period.

In each period, DM communicates with the expert about the state of that period before making a decision. The communication process is as follows. After observing $s_{t}$, the expert sends a report $m_{t} \in[0,1]$ to DM. We assume that the expert can send any report in $[0,1]$, which can be different from the state. Also, the expert does not incur any cost in sending a report. After receiving the report from the expert, DM updates her belief about the distribution of the state in that period and her belief about the expert's type. Then DM takes a decision $a_{t}$. The timeline of the game between the expert and DM is shown in Figure 1.

We assume that DM and the expert do not discount their future payoffs. The total payoff of DM is the sum of her payoffs in two periods, which is

$$
u_{\mathrm{DM}}\left(a_{1}, s_{1}, a_{2}, s_{2}\right)=-\left(a_{1}-s_{1}\right)^{2}-\left(a_{2}-s_{2}\right)^{2},
$$

and the total payoff of the expert is

$$
u_{E}\left(a_{1}, s_{1}, a_{2}, s_{2}\right)=-\left(a_{1}-s_{1}-\beta\right)^{2}-\left(a_{2}-s_{2}-\beta\right)^{2} .
$$

A strategy of the unbiased expert in period $t$ is a mapping from the state space $[0,1]$ to the space of possible reports $[0,1]$, denoted as $K_{t}$. A strategy of the biased expert is another mapping from the state space to the space of possible reports, denoted as $K_{t}^{\prime}$. A strategy of DM is a mapping from the space of possible reports to the space of decisions, denoted as $J_{t}$. A belief of DM about the state in period $t$ after receiving a report $m_{t}$ from the expert is a probability distribution of $s_{t}$ conditional on $m_{t}$, denoted as $F\left(s_{t} \mid m_{t}\right)$. A belief of DM about the type of the expert after receiving a report $m_{t}$ is 
a probability that the expert is unbiased given $m_{t}$, denoted as $\lambda\left(m_{t}\right)$.

The equilibrium concept that we use in this paper is perfect Bayesian Nash equilibrium (hereafter the equilibrium). An equilibrium consists of a strategy for each type of the expert, a strategy of DM, a belief of DM about the state, and a belief of DM about the type of the expert. To be an equilibrium, these strategies and beliefs must satisfy the following conditions: the strategy of each type of the expert maximizes the expert's expected payoff, given the strategy of DM; DM's strategy maximizes her expected payoff, given DM's beliefs about the state and the type of the expert; DM's beliefs about the state and the type of the expert are derived from the strategies of two types of the expert using Bayes' rule.

We first characterize an equilibrium in period two for an arbitrary belief of DM at the beginning of this period regarding the type of the expert. The proofs of formal results are relegated to the appendix.

Lemma 1. If DM believes at the beginning of period two that the expert is unbiased with probability $\lambda$ and the possible bias of the expert is not too large, in particular, $b \leq 1 / 4$, there exists an equilibrium in period two where each type of the expert sends two possible reports $m_{2}^{*}$ and $m_{2}^{\prime *}$. The biased (unbiased, resp.) expert sends report $m_{2}^{*}$ if and only if period-two state is less than $\bar{s}_{2 b}^{*}\left(\bar{s}_{2}^{*}\right)$. Otherwise, they send report $m_{2}^{\prime *}$. DM takes decision $a_{2}^{*}\left(a_{2}^{\prime *}\right)$ after receiving report $m_{2}^{*}\left(m_{2}^{\prime *}\right) \cdot a_{2}^{*}, a_{2}^{\prime *}, \bar{s}_{2 b}^{*}$, and $\bar{s}_{2}^{*}$ are given by

$$
\begin{aligned}
& a_{2}^{*}=\frac{1}{4}-(1-\lambda) b, \\
& a_{2}^{\prime *}=\frac{3}{4}-(1-\lambda) b, \\
& \bar{s}_{2 b}^{*}=\frac{1}{2}-(2-\lambda) b, \\
& \bar{s}_{2}^{*}=\frac{1}{2}-(1-\lambda) b .
\end{aligned}
$$

We can calculate the expected payoff of the biased expert in period two before he observes the state in period two. Since period-2 state is distributed uniformly over $[0,1]$, the state will be less than $\bar{s}_{2 b}^{*}$ with probability $\bar{s}_{2 b}^{*}$. In this case, the biased expert will send report $m_{2}^{*}$ and DM will take decision $a_{2}^{*}$. In addition, period-2 state will be higher than $\bar{s}_{2 b}^{*}$ with probability $1-\bar{s}_{2 b}^{*}$. In this case, the biased expert will send report $m_{2}^{\prime *}$ and DM will take decision $a_{2}^{\prime *}$. Therefore, the biased expert's expected payoff in period two before he observes the state in period two is

$$
\begin{aligned}
& \bar{s}_{2 b}^{*} E_{s_{2}}\left[-\left(a_{2}^{*}-s_{2}-b\right)^{2} \mid s_{2} \leq \bar{s}_{2 b}^{*}\right] \\
& \quad+\left(1-\bar{s}_{2 b}^{*}\right) E_{s_{2}}\left[-\left(a_{2}^{\prime *}-s_{2}-b\right)^{2} \mid s_{2} \geq \bar{s}_{2 b}^{*}\right] .
\end{aligned}
$$

By substituting $a_{2}^{*}, a_{2}^{\prime *}$, and $\bar{s}_{2 b}^{*}$ with their respective values, the biased expert's expected payoff in period two is

$$
-\frac{1}{2}(2-\lambda)^{2} b^{2}-\frac{1}{48} \text {. }
$$

Similarly, we can show that the unbiased expert's expected payoff in period two before the expert observes period-two state is

$$
-\frac{1}{2}(1-\lambda)^{2} b^{2}-\frac{1}{48}
$$

It is straightforward to see that the expected payoffs of biased and unbiased experts are both strictly increasing in $\lambda$ for $0 \leq \lambda \leq 1$. This implies that both types of the expert prefer DM to believe that the expert is unbiased with a higher probability at the beginning of period two. Therefore in period one, both types of the expert have incentives to manipulate the belief of DM about the type of the expert, in order to receive a higher expected payoff in period two.

Next, we consider the game between DM and the expert in period one. We have the following conclusion.

Proposition 2. When the possible bias of the expert is not too large, in particular, $b \leq 1 / 4$, there exists an equilibrium in period one. In equilibrium, there are two possible reports $m_{1}^{*}$ and $m_{1}^{\prime *}$ sent by each type of the expert. The biased (unbiased) expert sends report $m_{1}^{*}$ if and only if period-one state is less than $\bar{s}_{1 b}^{*}\left(\bar{s}_{1}^{*}\right)$. Otherwise, they send report $m_{1}^{\prime *}$. When receiving report $m_{1}^{*}\left(m_{1}^{\prime *}\right)$, DM's posterior belief about the type of the expert is $\lambda_{1}^{*}\left(\lambda_{1}^{\prime *}\right)$ and DM takes decision $a_{1}^{*}\left(a_{1}^{\prime *}\right) . m_{1}^{*}, m_{1}^{\prime *}$, $\bar{s}_{1 b}^{*}, \bar{s}_{1}^{*}, \lambda_{1}^{*}$, and $\lambda_{1}^{\prime *}$ are given by the solution to the following system of equations:

$$
\begin{aligned}
- & \left(a_{1}-\bar{s}_{1 b}-b\right)^{2}-\frac{1}{2}\left(2-\lambda_{1}\right)^{2} b^{2} \\
& =-\left(a_{1}^{\prime}-\bar{s}_{1 b}-b\right)^{2}-\frac{1}{2}\left(2-\lambda_{1}^{\prime}\right)^{2} b^{2}, \\
- & \left(a_{1}-\bar{s}_{1}\right)^{2}-\frac{1}{2}\left(1-\lambda_{1}\right)^{2} b^{2} \\
& =-\left(a_{1}^{\prime}-\bar{s}_{1}\right)^{2}-\frac{1}{2}\left(1-\lambda_{1}^{\prime}\right)^{2} b^{2}, \\
\lambda_{1} & =\frac{\bar{s}_{1}}{\bar{s}_{1}+\bar{s}_{1 b}}, \\
a_{1} & =\frac{1}{2} \frac{\bar{s}_{1}}{2}+\frac{1}{2} \frac{\bar{s}_{1 b}}{2}, \\
\lambda_{1}^{\prime} & =\frac{1-\bar{s}_{1}}{\left(1-\bar{s}_{1}\right)+\left(1-\bar{s}_{1 b}\right)}, \\
a_{1}^{\prime}= & \frac{1}{2} \frac{1+\bar{s}_{1}}{2}+\frac{1}{2} \frac{1+\bar{s}_{1 b}}{2} .
\end{aligned}
$$

In Figure 2, we depict variables $a_{1}^{*}, a_{1}^{\prime *}, \bar{s}_{1 b}^{*}, \bar{s}_{1}^{*}, \lambda_{1}^{*}$, and $\lambda_{1}^{\prime *}$ for different values of $b$.

\section{Comparison with No Reputational Concerns}

In this section, we consider the following question: whether the ex ante expected payoff of DM when the expert has reputational concerns is higher than that of DM when the expert does not have such concerns. In particular, we 


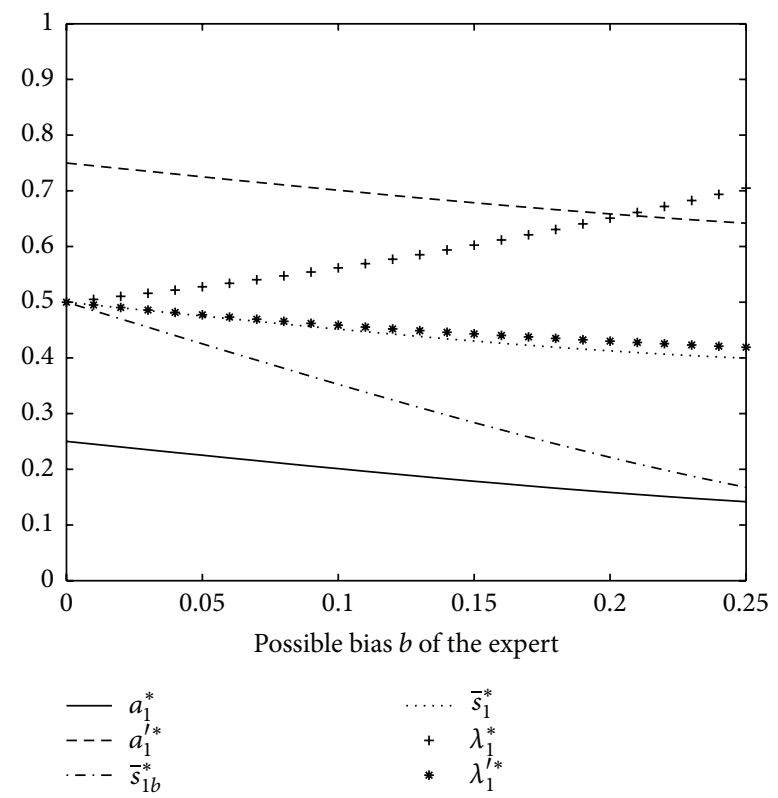

FIGURE 2: Equilibrium variables in period one for different possible biases of the expert.

compare the ex ante expected payoff of DM in period one of our model with that of DM in a model where the expert communicates with DM in only one period and therefore has no reputational concerns.

Consider a model where there is only one period and DM consults the expert about the state only once. Other aspects of the model are the same as the model in Section 2. Since there is only one period, DM's belief about the type of the expert at the end of the period does not affect the expected payoff of the expert. In this sense, the expert has no reputational concerns.

Note that the analysis of the model is identical to the analysis of period-two game between the expert and DM in Section 2, except that DM's belief about the expert's type before receiving any report from the expert is $1 / 2$, instead of $\lambda$ as in Section 2. By following identical arguments as in Section 2, we can characterize an equilibrium:

$$
\begin{aligned}
a_{1}^{n} & =\frac{1}{4}-\frac{1}{2 b}, \\
a_{1}^{\prime n} & =\frac{3}{4}-\frac{1}{2 b}, \\
\bar{s}_{1 b}^{n} & =\frac{1}{2}-\frac{3}{2 b}, \\
\bar{s}_{1}^{n} & =\frac{1}{2}-\frac{1}{2 b} .
\end{aligned}
$$

The ex ante expected payoff of DM before receiving the expert's report can be calculated as follows:

$$
\begin{aligned}
& \frac{1}{2}\left\{\bar{s}_{1 b}^{n} E_{s_{1}}\left[-\left(a_{1}^{n}-s_{1}\right)^{2} \mid s_{1} \leq \bar{s}_{1 b}^{n}\right]\right. \\
& \left.\quad+\left(1-\bar{s}_{1 b}^{n}\right) E_{s_{1}}\left[-\left(a_{1}^{\prime n}-s_{1}\right)^{2} \mid s_{1}>\bar{s}_{1 b}^{n}\right]\right\}
\end{aligned}
$$

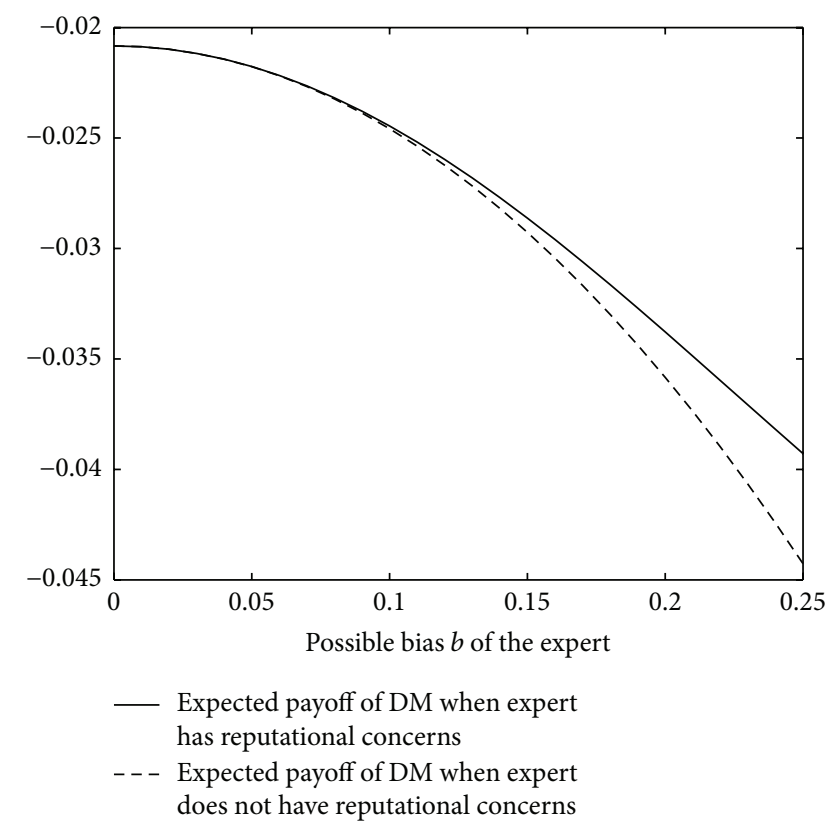

FIGURE 3: Ex ante expected payoff of DM when the expert is concerned with reputation versus that of DM when the expert does not have such concerns.

$$
\begin{aligned}
& +\frac{1}{2}\left\{\bar{s}_{1}^{n} E_{s_{1}}\left[-\left(a_{1}^{n}-s_{1}\right)^{2} \mid s_{1} \leq \bar{s}_{1}^{n}\right]\right. \\
& \left.+\left(1-\bar{s}_{1}^{n}\right) E_{s_{1}}\left[-\left(a_{1}^{\prime n}-s_{1}\right)^{2} \mid s_{1}>\bar{s}_{1}^{n}\right]\right\},
\end{aligned}
$$

which can be shown to be

$$
-\frac{3}{8} b^{2}-\frac{1}{48}
$$

By replacing variables $a_{1}^{n}, a_{1}^{\prime n}, \bar{s}_{1 b}^{n}$, and $\bar{s}_{1}^{n}$ by $a_{1}^{*}, a_{1}^{\prime *}, \bar{s}_{1 b}^{*}$, and $\bar{s}_{1}^{*}$, respectively, in (11), we can calculate the ex ante expected payoff of DM in period one of the model in Section 2 where the expert has reputational concerns.

In Figure 3, we draw the ex ante expected payoff of DM when the expert has reputational concerns and that of DM when the expert does not have such concerns. Both ex ante payoffs are drawn for different possible biases of the expert. From the figure, we can see that DM's ex ante expected payoff when the expert has reputational concerns is no less than that of DM when the expert does not have such concerns. When the possible bias of the expert is large, the former payoff is strictly greater than the latter one. This result indicates the beneficial effect of the expert's reputational concerns on the expected payoff of DM, especially when the expert can possibly have a large bias.

\section{Conclusion}

The current paper studies a mathematical model in which an uninformed decision maker consults an informed expert about the optimal decision. The expert may be biased in 
favoring a decision that is different from the ideal decision of the decision maker. It is shown that there is an equilibrium where the expert is concerned with his reputation for being unbiased, which is the probability that the expert is unbiased as perceived by the decision maker. Different from previous models where the expert is concerned with his reputation for being unbiased, the decision and state spaces are both continuous in the current paper. We show that, from an ex ante point of view, the decision maker's expected payoff is higher when the expert cares about his reputation than that of DM when the expert does not have reputational concerns.

The paper focuses on an equilibrium of the model where there are only two possible reports sent by the expert and two possible decisions made by the decision maker. The main reason for this is tractability. When the possible bias of the expert is small, we conjecture that there exists an equilibrium with more than two possible reports and more than two possible decisions. Characterizing such an equilibrium is more involved and is left for future research.

\section{Appendix}

\section{A. Proof of Lemma 1}

Suppose that, at the beginning of period two, DM believes that the expert is unbiased with probability $\lambda$. Consider the problem that the biased expert faces when observing that the period-two state is $s_{2}$. Suppose that the expert faces a choice between sending two possible reports denoted as $m_{2}$ and $m_{2}^{\prime}$. Suppose further that DM takes decision $a_{2}\left(a_{2}^{\prime}\right)$ when receiving report $m_{2}\left(m_{2}^{\prime}\right)$ from the expert and $a_{2}<a_{2}^{\prime}$. Therefore, the biased expert's second-period payoff is $-\left(a_{2}-\right.$ $\left.s_{2}-b\right)^{2}$ (resp., $\left.-\left(a_{2}^{\prime}-s_{2}-b\right)^{2}\right)$ by sending report $m_{2}$ (resp., $m_{2}^{\prime}$ ). It is straightforward to verify that the former payoff is strictly greater than the latter one if and only if $s_{2}$ is less than cutoff value $\bar{s}_{2 b}$, where

$$
\bar{s}_{2 b}=\frac{\left(a_{2}+a_{2}^{\prime}\right)}{2}-b .
$$

Similarly, the unbiased expert's period-two payoff by sending report $m_{2}$ is greater than that by sending report $m_{2}^{\prime}$, if and only if the second-period state $s_{2}$ is less than cutoff value $\bar{s}_{2}$, where

$$
\bar{s}_{2}=\frac{\left(a_{2}+a_{2}^{\prime}\right)}{2} .
$$

Given the strategies of biased and unbiased experts as described above, DM updates her belief about period-two state after receiving a report from the expert. After DM receives report $m_{2}$, DM will infer that, with probability $\lambda$, the expert is unbiased; therefore period-two state is less than $\bar{s}_{2}$, and with probability $1-\lambda$, the expert is biased; therefore period-two state is less than $\bar{s}_{2 b}$. Given DM's belief about the state after receiving report $m_{2}$, DM's optimal decision is equal to the expected value of period-two state. In other words,
$a_{2}=E_{s_{2}}\left[s_{2} \mid m_{2}\right]$. We have that DM's optimal decision when receiving report $m_{2}$ is

$$
a_{2}=\lambda \frac{\bar{s}_{2}}{2}+(1-\lambda) \frac{\bar{s}_{2 b}}{2} .
$$

In a similar fashion, we can find that after receiving report $m_{2}^{\prime}$ DM's best decision is

$$
a_{2}^{\prime}=\lambda \frac{1+\bar{s}_{2}}{2}+(1-\lambda) \frac{1+\bar{s}_{2 b}}{2} .
$$

From solving the above four equations, we can get the values of $a_{2}^{*}, a_{2}^{\prime *}, \bar{s}_{2}^{*}$, and $\bar{s}_{2 b}^{*}$ as stated in the lemma. In order for the above solution to represent an equilibrium in period two, cutoff states $\bar{s}_{2 b}^{*}$ and $\bar{s}_{2}^{*}$ must both lie between 0 and 1 . Therefore, the possible bias of the expert must not be too large; in particular, $b \leq 1 /(4-2 \lambda)$. We consider a situation where the possible bias of the expert is small enough such that an equilibrium as represented by the above solution exists even when DM believes that the expert is biased for sure $(\lambda=0)$. In order words, $b \leq 1 / 4$.

\section{B. Proof of Proposition 2}

Consider two possible decisions of DM in period one, $a_{1}$ and $a_{1}^{\prime}$, where $a_{1}<a_{1}^{\prime}$. Suppose that DM takes decision $a_{1}\left(a_{1}^{\prime}\right)$ when receiving report $m_{1}\left(m_{1}^{\prime}\right)$. In addition, suppose that DM believes that the expert is unbiased with probability $\lambda_{1}\left(\lambda_{1}^{\prime}\right)$ when receiving report $m_{1}\left(m_{1}^{\prime}\right)$.

Consider the problem of the biased expert when he observes that period-one state is $s_{1}$. If the expert sends report $m_{1}$, the expert's expected payoff, including the expected payoff in period two, is

$$
-\left(a_{1}-s_{1}-b\right)^{2}-\frac{1}{2}\left(2-\lambda_{1}\right)^{2} b^{2}-\frac{1}{48} .
$$

If the biased expert sends report $m_{1}^{\prime}$, the expert's expected payoff is

$$
-\left(a_{1}^{\prime}-s_{1}-b\right)^{2}-\frac{1}{2}\left(2-\lambda_{1}^{\prime}\right)^{2} b^{2}-\frac{1}{48} \text {. }
$$

It is straightforward to show that there exists a periodone state $\bar{s}_{1 b}$ satisfying that the expected payoff of the biased expert by sending report $m_{1}$ is equal to the expert's expected payoff by sending report $m_{2}$ when period-one state is $\bar{s}_{1 b}$. We have the following equation:

$$
\begin{aligned}
& -\left(a_{1}-\bar{s}_{1 b}-b\right)^{2}-\frac{1}{2}\left(2-\lambda_{1}\right)^{2} b^{2} \\
& =-\left(a_{1}^{\prime}-\bar{s}_{1 b}-b\right)^{2}-\frac{1}{2}\left(2-\lambda_{1}^{\prime}\right)^{2} b^{2} .
\end{aligned}
$$

In addition, the former payoff is higher than the latter payoff if and only if period-one state is less than $\bar{s}_{1 b}$. Similarly, there exists a period-one state $\bar{s}_{1}$ satisfying that the expected payoff of the unbiased expert by sending report $m_{1}$ is equal 
to the expert's expected payoff by sending report $m_{1}^{\prime}$ when period-one state is $\bar{s}_{1}$. We have that

$$
\begin{aligned}
& -\left(a_{1}-\bar{s}_{1}\right)^{2}-\frac{1}{2}\left(1-\lambda_{1}\right)^{2} b^{2} \\
& =-\left(a_{1}^{\prime}-\bar{s}_{1}\right)^{2}-\frac{1}{2}\left(1-\lambda_{1}^{\prime}\right)^{2} b^{2} .
\end{aligned}
$$

In addition, the former payoff is higher than the latter payoff if and only if period-one state is less than $\bar{s}_{1}$.

When receiving report $m_{1}$, by Bayes' rule, DM believes that the expert is unbiased with probability

$$
\lambda_{1}=\frac{\bar{s}_{1}}{\bar{s}_{1}+\bar{s}_{1 b}},
$$

and DM's optimal decision is

$$
a_{1}=E_{s_{1}}\left[s_{1} \mid m_{1}\right]=\frac{1}{2} \frac{\bar{s}_{1}}{2}+\frac{1}{2} \frac{\bar{s}_{1 b}}{2} .
$$

In a similar fashion, when receiving report $m_{1}^{\prime}, \mathrm{DM}$ believes that the expert is unbiased with probability

$$
\lambda_{1}^{\prime}=\frac{1-\bar{s}_{1}}{\left(1-\bar{s}_{1}\right)+\left(1-\bar{s}_{1 b}\right)},
$$

and DM's optimal decision when receiving report $m_{1}^{\prime}$ is

$$
a_{1}^{\prime}=E_{s_{1}}\left[s_{1} \mid m_{1}^{\prime}\right]=\frac{1}{2} \frac{1+\bar{s}_{1}}{2}+\frac{1}{2} \frac{1+\bar{s}_{1 b}}{2} .
$$

\section{Competing Interests}

The authors declare that they have no competing interests.

\section{Acknowledgments}

This research is supported by the Fundamental Research Funds for the Central Universities (nos. JBK151123 and JBK160102).

\section{References}

[1] M. Ottaviani and P. N. Sørensen, "Reputational cheap talk," The RAND Journal of Economics, vol. 37, no. 1, pp. 155-175, 2006.

[2] S. Bourjade and B. Jullien, "The roles of reputation and transparency on the behavior of biased experts," RAND Journal of Economics, vol. 42, no. 3, pp. 575-594, 2011.

[3] J. Sobel, "A theory of credibility," Review of Economic Studies, vol. 52, no. 4, pp. 557-573, 1985.

[4] R. Benabou and G. Laroque, "Using privileged information to manipulate markets: insiders, gurus, and credibility," The Quarterly Journal of Economics, vol. 107, no. 3, pp. 921-958, 1992.

[5] W. Li, "Peddling influence through intermediaries," American Economic Review, vol. 100, no. 3, pp. 1136-1162, 2010.

[6] S. Morris, "Political correctness," Journal of Political Economy, vol. 109, no. 2, pp. 231-265, 2001. 


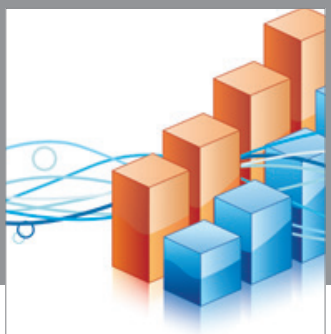

Advances in

Operations Research

vatem alat4

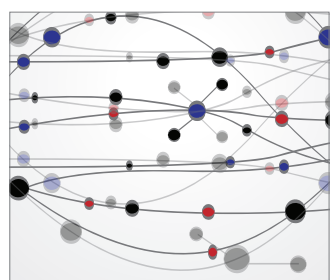

\section{The Scientific} World Journal
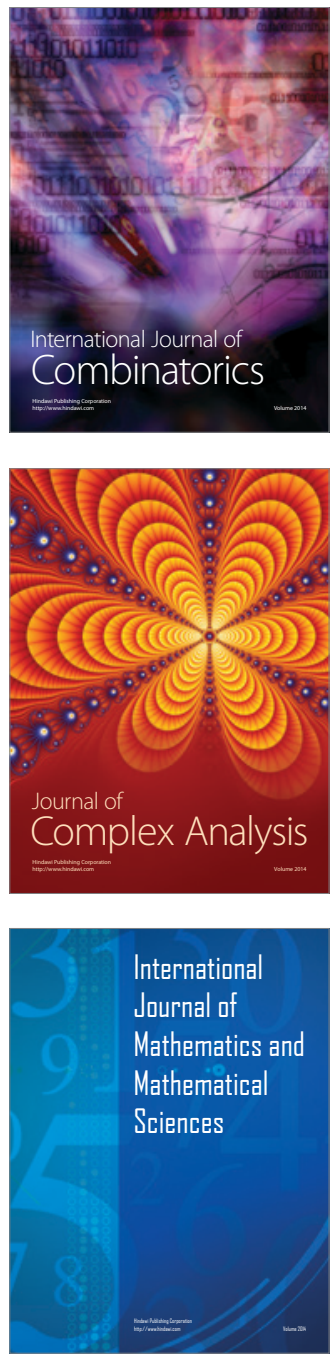
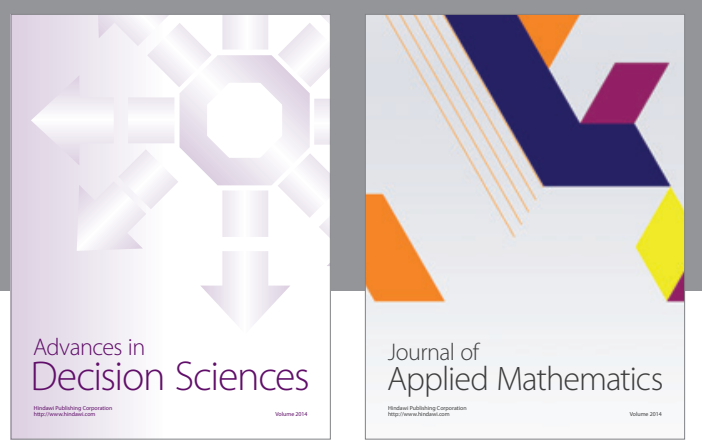

Algebra

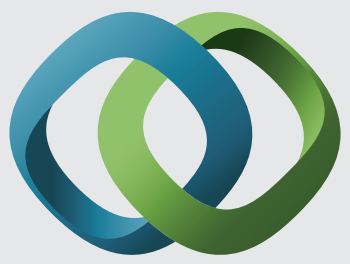

\section{Hindawi}

Submit your manuscripts at

http://www.hindawi.com
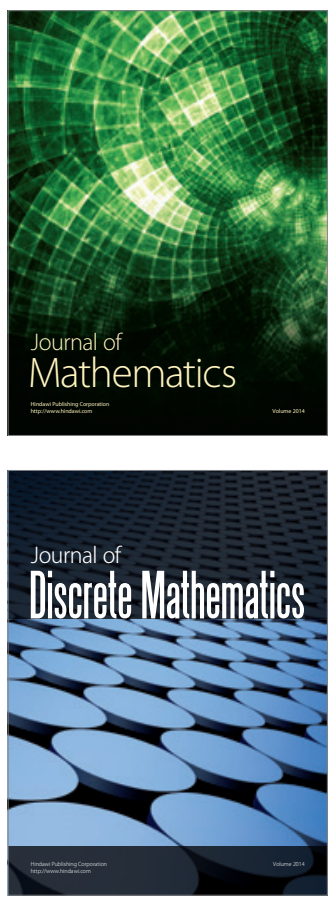

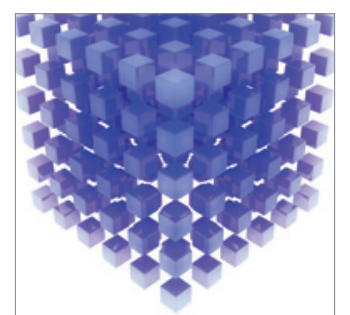

Mathematical Problems in Engineering
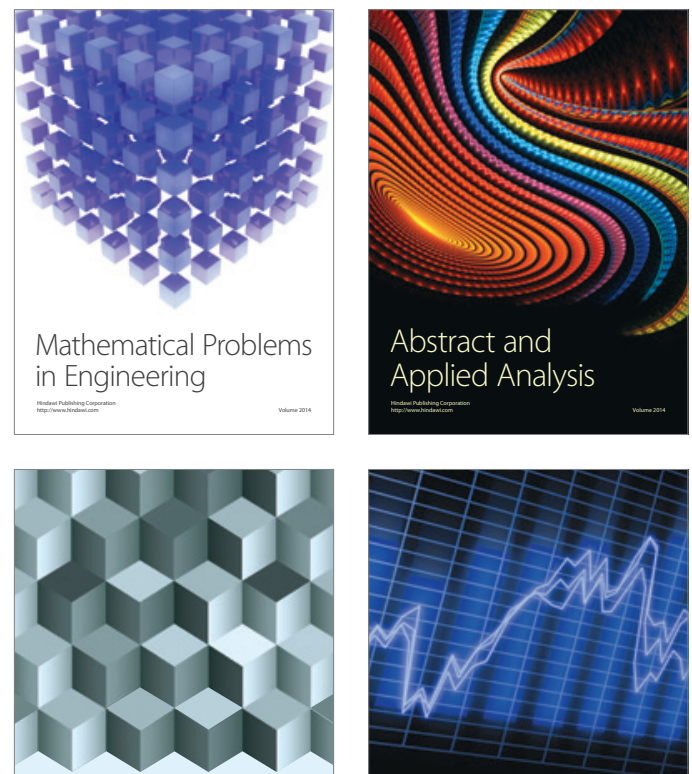

Journal of

Function Spaces

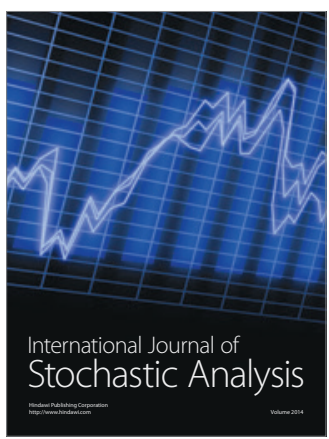

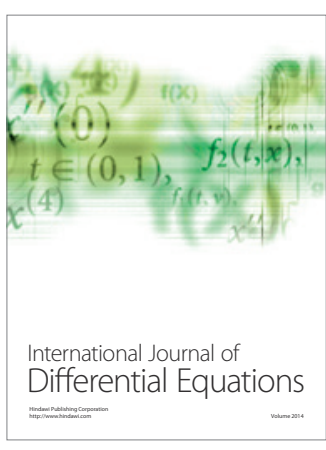
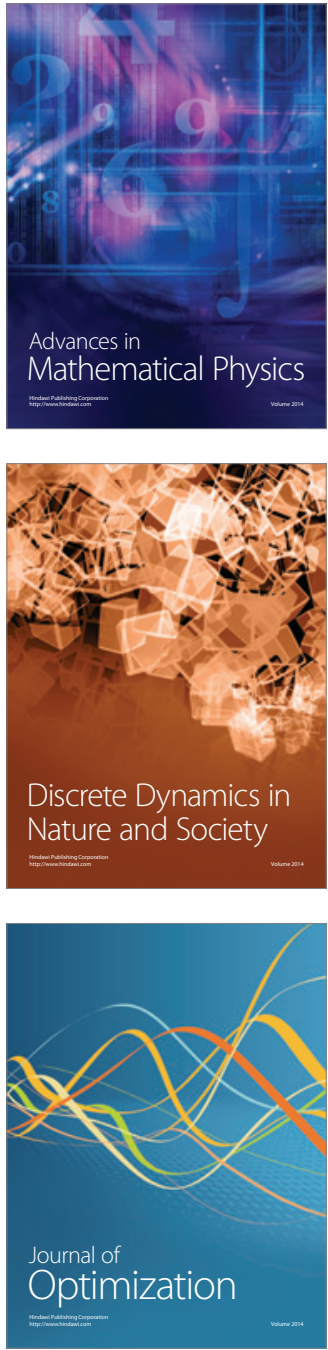